\title{
Tanshinone I induces apoptosis and protective autophagy in human glioblastoma cells via a reactive oxygen species-dependent pathway
}

\author{
SHANGGUAN JIAN $^{1 *}$, LIANG CHEN $^{1 *}$, LIAN MINXUE ${ }^{1}$, CHE HONGMIN $^{2}$, TANG RONGHUA $^{3}$, \\ FAN XIAOXUAN $^{1}$, ZHANG BINBIN $^{1}$ and GUO SHIWEN ${ }^{1}$ \\ ${ }^{1}$ Department of Neurosurgery, The First Affiliated Hospital of Xi'an Jiaotong University; \\ ${ }^{2}$ Department of Neurosurgery, Xi'an Gaoxin Hospital, Xi'an, Shaanxi 710061; ${ }^{3}$ Department of Neurosurgery, \\ Chongqing University Cancer Hospital, Chongqing 400030, P.R. China
}

Received May 11, 2019; Accepted November 25, 2019

DOI: $10.3892 / \mathrm{ijmm} .2020 .4499$

\begin{abstract}
Glioma is the most common primary malignancy of the central nervous system and is associated with high mortality rates. Despite the available treatment options including surgery, radiotherapy and chemotherapy, the median patient survival rate is low. Therefore, the development of novel anticancer agents for the treatment of glioma is urgently required. Tanshinone I (TS I) is a tanshinone compound that is isolated from Danshen. Accumulating evidence indicates that TS I exhibits antiproliferative activity in a variety of cancer types. However, the role of TS I and its mechanism of action in human glioma remain to be elucidated. In the present study, the anticancer potential of TS I against human glioma U87 MG cells was investigated. The results indicated that TS I exerted a potential cytotoxic effect on human glioma U87 MG cells. TS I was found to induce cell proliferation, inhibition, cell cycle arrest, apoptosis and autophagy in U87 MG cells. Mechanistic experiments indicated that TS I activated endoplasmic reticulum (ER) stress and inhibited AKT signaling and apoptosis in human glioma U87 MG cells. Furthermore, the present study demonstrated that TS I induced protective autophagy in U87 MG cells. Additionally, ER stress and AKT signal-mediated apoptosis and protective autophagy were found to be induced by TS I via intracellular reactive oxygen species accumulation. The results of the present study demonstrated that TS I may be a potential anticancer drug candidate that may be of value in the treatment of human glioma.
\end{abstract}

Correspondence to: Professor Guo Shiwen, Department of Neurosurgery, The First Affiliated Hospital of Xi'an Jiaotong University, 277 Yanta West Road, Xi'an, Shaanxi 710061, P.R. China E-mail: gsw1962@126.com

*Contributed equally

Key words: tanshinone I, U87 MG cells, apoptosis, cell cycle arrest, endoplasmic reticulum stress, reactive oxygen species, autophagy, AKT

\section{Introduction}

It has been well established that malignant glioma is one of the most common types of brain neoplasms, and is associated with high mortality and poor 5-year survival rates worldwide (1). The currently available treatment for glioma involves surgical removal of the tumor, chemotherapy and radiotherapy, which can significantly improve the survival rates of the patients (2). However, for the majority of patients with glioma, prognosis is poor, with a median survival of $\sim 14$ months (3). Temozolomide (TMZ) is the most effective chemotherapeutic agent for glioma treatment (3). However, the response rate to TMZ treatment is accompanied by resistance to chemotherapy and high rates of toxicity, which may lead to patients experiencing severe nausea, vomiting and fetotoxic effects (4). Therefore, the development of novel anticancer agents that may be used to decrease glioma progression requires further investigation.

Danshen (Salvia miltiorrhiza Bunge) is a traditional Chinese herb that has been successfully used for the treatment of cardiovascular disease in Asian countries $(5,6)$. TS I has been demonstrated to be one of the bioactive components of Danshen, and has been reported to possess antioxidant, anti-inflammatory and anticancer properties (7). Recent studies on TS I have focused on its anticancer activity (8-10). These results have demonstrated that TS I may induce the apoptosis of cancer cells in gastric $(10)$, human breast $(11,12)$ and human colon cancer $(13,14)$. However, to the best of our knowledge, the exact mechanisms underlying the effects of TS I on human glioma have not yet been determined.

To determine the mechanisms underlying the anticancer activity exhibited by TS I in human glioma, the present study was performed to elucidate the biological mechanisms through which TS I may induce the inhibition of human glioma U87 MG cell growth.

\section{Materials and methods}

Reagents and antibodies. TS I was purchased from Sigma-Aldrich; Merck KGaA. The anti-p-AKT (cat. no. 4058), anti-AKT (cat. no. 9272), anti-cleaved poly(ADP-ribose) 
polymerase (PARP) (cat. no. 5625), anti-GADPH (cat. no. 2118), anti-cyclin B1 (cat. no. 4138), anti-B-cell lymphoma (Bcl)-2 (cat. no. 15071), anti-beclin-1 (cat. no. 3738), anti-C/EBP homologous protein (CHOP) (cat. no. 2895), anti-p-eukaryotic initiation factor (eIF) $2 \alpha$ (Ser51) (cat. no. 9721), anti-eIF2 $\alpha$ (cat. no. 9722), anti-LC3B (cat. no. 2775) and anti-Bcl-2-associated X protein (Bax) (cat. no. 2774) antibodies were purchased from Cell Signaling Technology, Inc. The anti-p21 antibody (cat. no. MAB1047) was purchased from R\&D Systems, Inc. LY294002 was purchased from Merck KGaA. The Annexin V-FITC and propidium iodide (PI) kit was purchased from BD Biosciences; Becton, Dickinson and Company. $\mathrm{N}$-acetyl-L-cysteine (NAC), a reactive oxygen species (ROS) scavenger and 3-methyladenine (3-MA; an inhibitor of autophagy) were purchased from MedChem Express LLC.

Cell culture. The U87 MG glioma cell line was purchased from Procell Life Science \& Technology Co., Ltd. (cat no. CL-0238). The cell line was established in the University of Uppsala and was authenticated using STR profiling. Cells were maintained in DMEM supplemented with 10\% FBS (Procell) and $1 \mathrm{X}$ penicillin-streptomycin solution.

Cell viability assay. U87 MG glioma cell viability was measured using a Cell Counting Kit-8 (CCK-8) assay. U87 MG cells were then seeded into a 96 -well plate $\left(6 \times 10^{3}\right.$ cells/well) for $24 \mathrm{~h}$. Cells were then treated with TS I $(0,0.625,1.25,2.5$, 5 or $10 \mu \mathrm{M}$ ), and incubated for an additional $24 \mathrm{~h}$. A total of $10 \mu \mathrm{l}$ CCK- 8 solution was subsequently added to each well and cells were then incubated for $4 \mathrm{~h}$. The absorbance value of each well was measured using an ELISA reader at a wavelength of $450 \mathrm{~nm}$. All experiments were performed three times, and results are expressed as the mean \pm standard deviation.

Cell cycle assay. The cell cycle distribution of human glioma U87 MG cells was analyzed using flow cytometry and PI staining. Cells were seeded $\left(1 \times 10^{6}\right.$ cells/well) into 6-well plates and treated with different TS I concentrations $(0,0.625$, 1.25 and $2.5 \mu \mathrm{M}$ ) for $24 \mathrm{~h}$. The treated cell groups were then fixed in cold $70 \%$ ethanol for $2 \mathrm{~h}$. RNase A $(60 \mu \mathrm{g} / \mathrm{ml})$ and PI $(50 \mu \mathrm{g} / \mathrm{ml})$ in PBS were added, and samples were incubated for $30 \mathrm{~min}$ in the dark at room temperature. The cell cycle distribution was analyzed using flow cytometry (FACSCalibur; BD Biosciences; Becton, Dickinson and Company). A total of 10,000 cells were collected for each cell group. The percentage of cell populations at subG0/G1, G2/M and $\mathrm{S}$ phases were examined using Modfit LT version 2.0 software (Verity Software House). Three independent experiments were performed.

Cell apoptosis assay. Cell apoptosis was assessed using an Annexin V-FITC/PI kit (BD Pharmingen; BD Biosciences) and the apoptotic rate was analyzed using a flow cytometer (FACSCalibur; BD Biosciences; Becton, Dickinson and Company). U87 MG cells were seeded $\left(1 \times 10^{6}\right.$ cells/well) into 6 -well plates and treated with different TS I concentrations $(0,0.625,1.25$ and $2.5 \mu \mathrm{M})$ for $24 \mathrm{~h}$. Cells were then suspended in a 5-ml culture tube with $1 \mathrm{X}$ binding buffer (provided with the Annexin V-FITC/PI kit) at a density of $1 \times 10^{6}$ cells $/ \mathrm{ml}$, stained using Annexin V-FITC and counterstained using PI in binding buffer at room temperature for $15 \mathrm{~min}$. The number of apoptotic cells was then determined using a flow cytometer (FACSCalibur; BD Biosciences; Becton, Dickinson and Company).

Western blot analysis. Cells were seeded onto $35-\mathrm{mm}$ plates $\left(2 \times 10^{6}\right.$ cells), with $2 \mathrm{ml}$ complete DMEM for $24 \mathrm{~h}$. Following TS I treatment, cells were washed with PBS, and lysed with RIPA lysis buffer $(150 \mathrm{mmol} / \mathrm{l} ; \mathrm{NaCl} 50 \mathrm{mmol} / \mathrm{l}$ Tris-HCl; pH 7.4; $1 \%$ Triton X-100; $1 \%$ sodium deoxycholate; $0.1 \%$ SDS) with $1 \mathrm{mM}$ sodium orthovanadate, $1 \mathrm{mM}$ MPMSF and $1 \%$ cocktail of protease inhibitors (Sigma-Aldrich; Merck KGaA). Protein concentration was determined using the BCA method. Equal quantities of protein (40 $\mu \mathrm{g} / \mathrm{lane})$ were separated using 6-12\% SDS-PAGE and transferred onto nitrocellulose membranes (EMD Millipore). The membranes were blocked with $5 \%$ non-fat milk for $2 \mathrm{~h}$ at $25^{\circ} \mathrm{C}$. The membranes were incubated with a variety of primary antibodies as follows: AKT $(1: 1,000)$, p-AKT $(1: 1,000)$, cyclin B1 $(1: 1,000)$, cleaved PARP $(1: 1,000), \mathrm{Bcl}-2(1: 1,000)$, Bax $(1: 1,000)$ or p21 $(1: 1,000)$, beclin-1 $(1: 2,000)$, CHOP $(1: 1,000)$, p-eIF2 $\alpha(\operatorname{Ser} 51)(1: 3,000), \operatorname{eIF} 2 \alpha(1: 4,000)$ and LC3B $(1: 2,000)$, followed by incubation with anti-rabbit HRP secondary antibody (1:20,000, cat. no. 7074, Cell Signaling Technology, Inc.) or anti-mouse HRP-conjugated secondary antibody $(1: 20,000$, cat. no. 7076, Cell Signaling Technology, Inc.) for $2 \mathrm{~h}$ at $25^{\circ} \mathrm{C}$. GAPDH $(1: 3,000)$ served as a loading control. Visualization was achieved using SuperSignal West Pico chemiluminescent Substrate (Pierce; Thermo Fisher Scientific, Inc.).

Measurement of ROS generation. Intracellular ROS levels were determined using a ROS assay kit (Keygen Biotech Co., Ltd.). Briefly, the cells were plated in 6-well plates at a density of $2.0 \times 10^{5}$ cells/well for $24 \mathrm{~h}$. Following treatment with TS I $(0,0.625,1.25$ and $2.5 \mu \mathrm{M})$ for $12 \mathrm{~h}$, cells were then incubated with $10 \mu \mathrm{M}$ DCFH-DA for $15 \mathrm{~min}$ at $37^{\circ} \mathrm{C}$ in the dark. Next, the cells were examined by flow cytometry using a FACScalibur Flow Cytometer (BD Biosciences) and the data were analyzed by FlowJo 7.6 software (FlowJo LLC).

Statistical analysis. All data are presented as the mean \pm standard deviation. A Student's t-test and one-way ANOVA followed by Dunnett's post hoc test were used to determine significance for differences among groups. $\mathrm{P}<0.05$ was considered to indicate a statistically significant difference.

\section{Result}

TS I exerts potent cytotoxic effects on human glioma U87 MG cells. To investigate the potent anticancer effects of TS I on U87 MG cells, cell viability was analyzed using a CCK-8 assay. In the present study, U87 MG cells were treated with TS I at different concentrations $(0,0.625,1.25,2.5,5$ and $10 \mu \mathrm{M})$ for $24 \mathrm{~h}$. The results demonstrated that TS I significantly inhibited U87 MG cell proliferation in a dose-dependent manner. Cell viability was reduced by $26.92 \pm 1.06,42.37 \pm 4.38$, $51.17 \pm 2.25$ and $66.39 \pm 2.24 \%$ at $1.25,2.5,5$ and $10 \mu \mathrm{M}$ TS I, respectively, after $24 \mathrm{~h}$ (Fig. 1). The $\mathrm{IC}_{50}$ value was found to be $3.35 \pm 0.44 \mu \mathrm{M}$ at $24 \mathrm{~h}$. The results demonstrated that TS I exerted marked cytotoxic effect on U87 MG cells by inhibiting cell proliferation. 
TS I induces cell cycle arrest at the G2/M phase in human glioma U87 MG cells. To investigate the mechanisms through which TS I inhibited U87 MG cell growth, flow cytometry was used to analyze the distribution of cells within the cell cycle following treatment with $0,0.625,1.25$ and $2.5 \mu \mathrm{M}$ TS I for $24 \mathrm{~h}$. Flow cytometry indicated that TS I significantly increased the percentage of cells in the $\mathrm{G} 2 / \mathrm{M}$ phase in a dose-dependent manner $(6.61 \pm 0.40,11.84 \pm 1.08$ and $15.79 \pm 1.79 \%$ in the 0.625 , 1.25 and $2.5 \mu \mathrm{M}$ treatment groups, respectively) compared with the control group (5.82 $\pm 0.67 \%)$ (Fig. 2A). Additionally, TS I treatment significantly decreased the expression of cyclin B1 and increased the expression of p21 (Fig. 2B). These results indicated that the downregulation of cyclin B1 expression and the upregulation of p21 expression contributed to TS I-induced G2/M phase arrest and antiproliferation effect in U87 MG cells.

TS I induces apoptosis in human glioma U87 MG cells. The present study assessed whether the inhibition of TS I-induced U87 MG cell growth was associated with apoptosis. Flow cytometry, which was performed using Annexin V-PI staining, demonstrated that, following treatment with TS I for $24 \mathrm{~h}$, the proportion of apoptotic U87 MG cells was markedly increased in a concentration-dependent manner. The percentage of apoptotic cells was $6.34 \pm 1.54,10.57 \pm 2.28,21.26 \pm 2.48$ and $30.33 \pm 3.13 \%$ in the $0,0.625,1.25$ and $2.5 \mu \mathrm{M}$ TS I groups, respectively (Fig. 3A). The levels of apoptosis-associated proteins were also determined using western blot analysis. As shown in Fig. 3B, TS I treatment decreased Bcl-2 and increased the cleaved PARP and Bax expression in a dose-dependent manner. These results suggested that TS I induces U87 MG cell apoptosis via the downregulation of $\mathrm{Bcl}-2$ expression and the upregulation of Bax and cleaved PARP expression.

TS I induces apoptosis via ROS production in human glioma U87 MG cells. TS I has been previously shown to induce apoptosis by increasing ROS levels in colon cancer cells (9). Therefore, whether ROS production was associated with TS I-induced apoptosis of U87 MG cells was assessed in the present study. Cells were treated with TS I $(0,0.625,1.25$ and $2.5 \mu \mathrm{M}$ ) for $12 \mathrm{~h}$ and analyzed using flow cytometry. As shown in Fig. 4A, ROS generation was significantly increased in a dose-dependent manner. Furthermore, cells were pre-treated with $4 \mathrm{mM}$ ROS scavenger NAC for $1 \mathrm{~h}$, and exposed to TS I $(1.25 \mu \mathrm{M})$ for $24 \mathrm{~h}$. Cell viability was analyzed using a CCK-8 assay. The results indicated that $4 \mathrm{mM}$ NAC significantly reduced TS I-induced inhibition of cell growth (Fig. 4B). Furthermore, pretreatment with NAC significantly restored the TS I-induced upregulation of Bax and cleaved PARP expression, and the downregulation of Bcl-2 expression (Fig. 4C). These data suggest that TS I promotes ROS generation and this effect is associated with TS I-induced cell apoptosis.

TS I induces apoptosis by suppressing the AKT signaling pathway in human glioma U87 MG cells. AKT signaling is a key downstream effector of phosphoinositide 3-kinase (PI3K) that regulates a variety of biological processes, including cell cycle progression, proliferation and apoptosis in cancer cells $(15,16)$ Previous studies have focused on the role of the AKT signaling pathway in human gliomas $(17,18)$. To determine the molecular

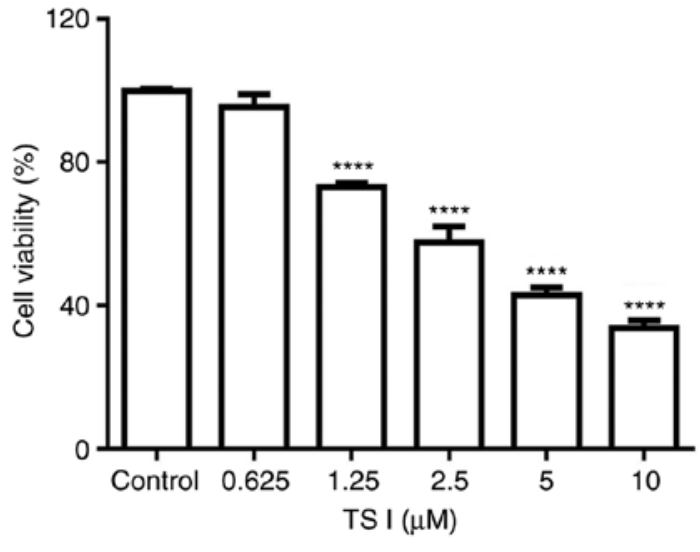

Figure 1. TS I inhibits the proliferation of U87 MG cells. The growth inhibitory effect of TS I was measured using a Cell Counting Kit-8 assay. Cell viability was examined after treatment with TS I $(0,0.625,1.25,2.5,5$ and $10 \mu \mathrm{m})$ for $24 \mathrm{~h}$. Data are presented as the mean \pm standard deviation of three separate experiments. ${ }^{* * * *} \mathrm{P}<0.0001$. TS-I, tanshinone I.

mechanisms underlying the TS I-induced apoptosis of U87 MG cells, the phosphorylation/activation of AKT in cells treated with TS I $(0,0.625,1.25$ and $2.5 \mu \mathrm{M})$ was assessed for $12 \mathrm{~h}$. The phosphorylation of AKT was found to decrease following TS I treatment in a dose-dependent manner. However, TS I exerted no effect on total AKT expression in U87 MG cells (Fig. 5A). To further elucidate whether TS I-induced apoptosis was associated with AKT signaling, cells were treated with $5 \mu \mathrm{M}$ PI3K inhibitor (LY294002) for $1 \mathrm{~h}$ prior to treatment with TS I $(1.25 \mu \mathrm{M})$ for $24 \mathrm{~h}$. Cell viability was measured in U87 MG cells treated with TS I alone or in combination with LY294002. As shown in Fig. 5B, TS I (1.25 $\mu \mathrm{M})$ in combination with $5 \mu \mathrm{M}$ LY294002 significantly inhibited U87 MG cell proliferation compared with TS I treatment alone. Additionally, apoptosis-associated protein expression was also determined using western blot analysis. As shown in Fig. 5C, LY294002 with TS I significantly decreased Bcl-2 protein expression and significantly increased the cleaved PARP and Bax protein expression compared with TS I treatment alone. Furthermore, the ROS-mediated AKT signaling pathway has been reported to be an important regulator of apoptosis in cancer cells, and has been shown to affect cell proliferation $(19,20)$. To elucidate the role of ROS production in the TS I-mediated inhibition of the AKT signaling pathway in U87 MG cells, the cells were treated with NAC for $1 \mathrm{~h}$ and subsequently treated with TS I $(1.25 \mu \mathrm{M})$ for $12 \mathrm{~h}$. As shown in Fig. 5D, western blot analysis revealed that TS I-induced reduction of p-AKT was reversed in the NAC pre-treatment group compared with the TS I alone group. These data indicated that TS I may induce apoptosis by regulating the ROS/AKT pathway in human glioma U87 MG cells.

TS I induces apoptosis via the ROS-mediated endoplasmic reticulum (ER) stress pathway in human glioma U87 MG cells. It has been previously reported that increased ROS generation can increase the expression of unfolded proteins and activate the ER stress response $(19,21)$. Therefore, in the present study, the expression of the ER stress-associated proteins p-eIF $2 \alpha$, eIF $2 \alpha$, activating transcription factor (ATF) 4 and CHOP was examined using western blot analysis. As shown in Fig. 6A, 


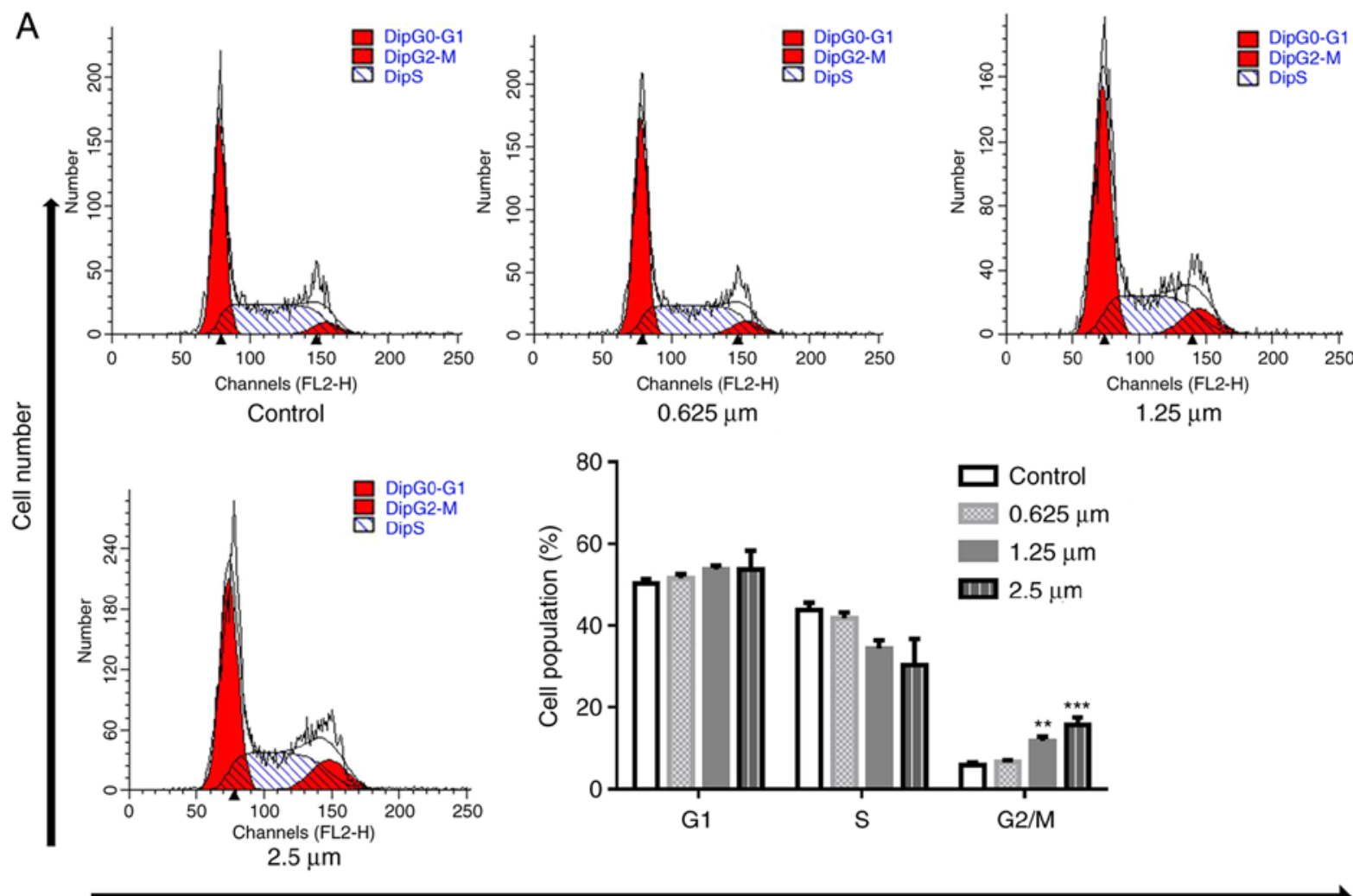

DNA content

B

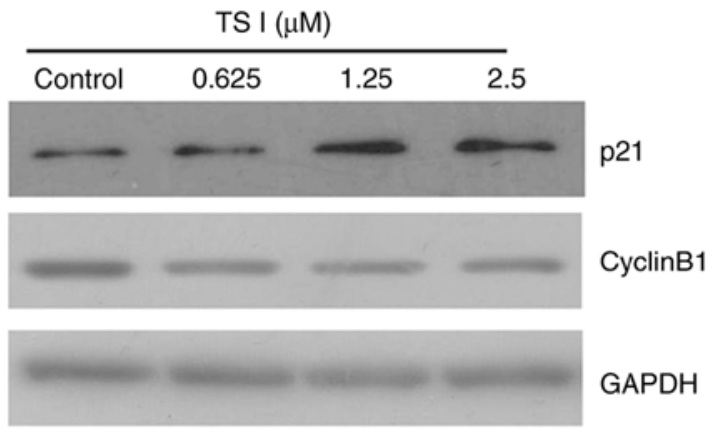

Figure 2. Effects of TS I on cell cycle distribution in U87 MG cells and the expression of cell cycle-associated proteins. (A) Cells were treated with TS I (0, $0.625,1.25$ and $2.5 \mu \mathrm{m}$ ) for $24 \mathrm{~h}$, and cell cycle distribution was analyzed using a flow cytometer. (B) Cells were treated with TS I $(0,0.625,1.25$ and $2.5 \mu \mathrm{m})$ for $12 \mathrm{~h}$, and the protein levels of cyclin B1 and p21 were assessed using western blot analysis in U87 MG cells. GAPDH was used as a loading control. ${ }^{* *} \mathrm{P}<0.01$; ${ }^{* * * *} \mathrm{P}<0.001$. TS-I, tanshinone I.

TS I significantly activated ER stress. To further verify the role of ROS production in the TS I-mediated activation of ER stress, cells were treated with $4 \mathrm{mM}$ NAC for $1 \mathrm{~h}$ and subsequently treated with TS I $(1.25 \mu \mathrm{M})$ for $12 \mathrm{~h}$. The results indicated that $4 \mathrm{mM}$ NAC pretreatment significantly attenuated TS I-mediated activation of ER stress (Fig. 6B). These results demonstrated that TS I may induce apoptosis via the ROS-mediated ER stress pathway in human glioma U87 MG cells.

TS I induces autophagy via ROS production in human glioma U87 MG cells. It has previously been suggested that TS I can induce autophagy in BGC823 and SGC7901 cells (10). Therefore, the present study assessed whether TS I induced autophagy in U87 MG cells. The expression of LC3B and beclin-1 was determined using western blot analysis. The results demonstrated that the expression of LC3B-II and beclin-1 were markedly increased in U87 MG cells following treatment with TS I (Fig. 7A). To further determine the role of autophagy in TS I-induced cell apoptosis, cells were treated with $5 \mathrm{mM} 3$-MA for $1 \mathrm{~h}$ and subsequently treated with TS I $(1.25 \mu \mathrm{M})$ for $24 \mathrm{~h}$. Cell viability was analyzed using a CCK-8 assay. As shown in Fig. 6B, co-treatment with $5 \mathrm{mM}$ 3-MA and TS I significantly enhanced the TS I-induced growth suppression of U87 MG cells (Fig. 7B). Additionally, increasing evidence has suggested that autophagy and ROS accumulation are closely associated events in a variety of cancer types (22-24). To elucidate the association between ROS production and TS I-mediated autophagy, cells were treated with $4 \mathrm{mM}$ NAC for $1 \mathrm{~h}$ and subsequently treated with TS I $(1.25 \mu \mathrm{M})$ for $12 \mathrm{~h}$. LC3B and beclin-1 expression was determined using western blot analysis. The results of the 
A
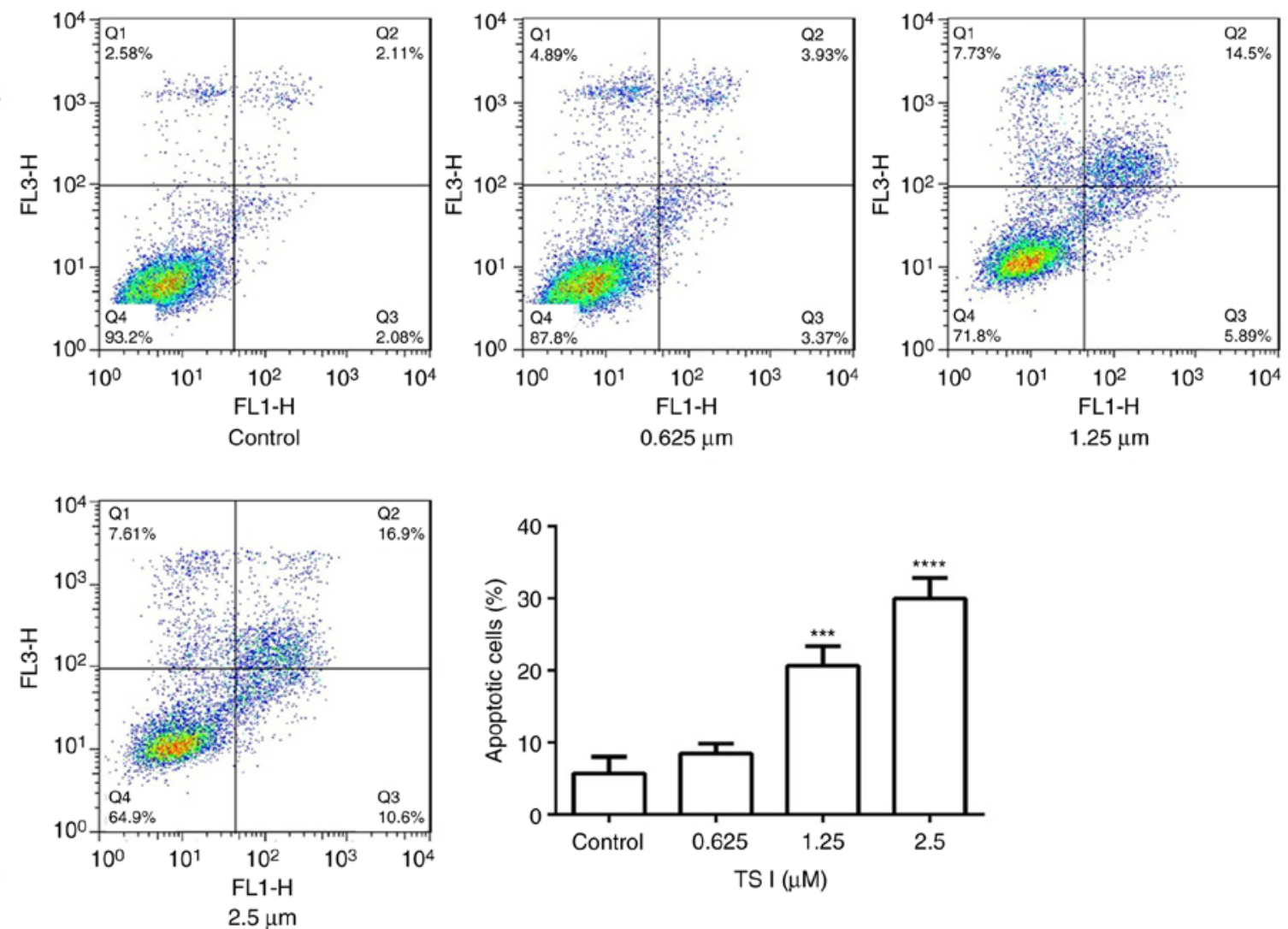

Annexin V

B
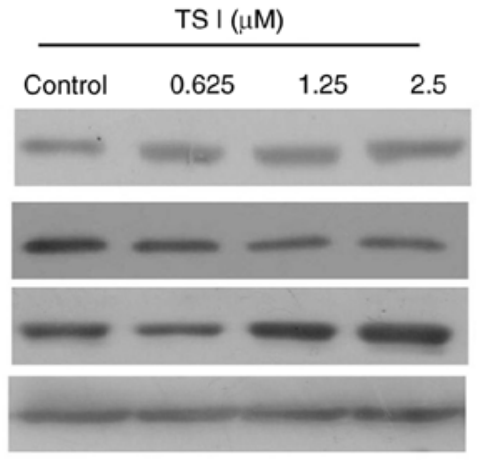

Bax

$\mathrm{Bcl}-2$

Cleaved PARP

GAPDH

Figure 3. Effects of TS I on U87 MG cell apoptosis and the expression of cell apoptosis-associated proteins. (A) Cells were treated with TS I $(0,0.625,1.25$ and $2.5 \mu \mathrm{m})$ for $24 \mathrm{~h}$, and cell apoptosis was analyzed using flow cytometry in U87 MG cells. (B) Cells were treated with TS I $(0,0.625,1.25$ and $2.5 \mu \mathrm{m})$ for $12 \mathrm{~h}$, and the protein levels of Bcl-2, Bax and cleaved PARP were assessed using western blot analysis in U87 MG cells. GAPDH was used as a loading control.

${ }^{* * *} \mathrm{P}<0.001 ;{ }^{* * * *} \mathrm{P}<0.0001$. TS-I, tanshinone I; Bcl-2, B-cell lymphoma 2; Bax, Bcl-2-associated X protein; PARP, poly(ADP-ribose) polymerase.

present study indicated that NAC pretreatment significantly attenuated LC3B-II and beclin-1 expression compared with TS I treatment alone (Fig. 7C). Therefore, these findings suggest that TS I may promote protective autophagy via ROS generation in U87 MG cells.

\section{Discussion}

TS I has been demonstrated to exert antiproliferative effects on gastric cancers cell lines (including BGC823 and SGC7901) (10), human breast cancer cell lines (including MCF-7 and MDA-MB-453) $(11,12)$ and human colon cancer cells $(13,14)$. However, the effect of TS I on human glioma has not been extensively investigated. The present study investigated the anticancer effects of TS I on human glioma U87 MG cells. The data demonstrated that TS I inhibited cell proliferation, induced G2/M phase arrest and triggered apoptosis in a dose-dependent manner. The underlying molecular mechanism may involve increased ROS production, decreased phosphorylation of AKT, activated autophagy, or an activated ER stress pathway in human glioma U87 MG cells. TS I was indicated to induce apoptosis via activating the ER stress pathway and inhibiting AKT signaling, and was shown to induce protective autophagy via ROS production in U87 MG cells.

It is well established that abnormal cell cycle progression may cause uncontrolled growth, and that cell cycle arrest can inhibit the proliferation and induce apoptosis in cancer 
A
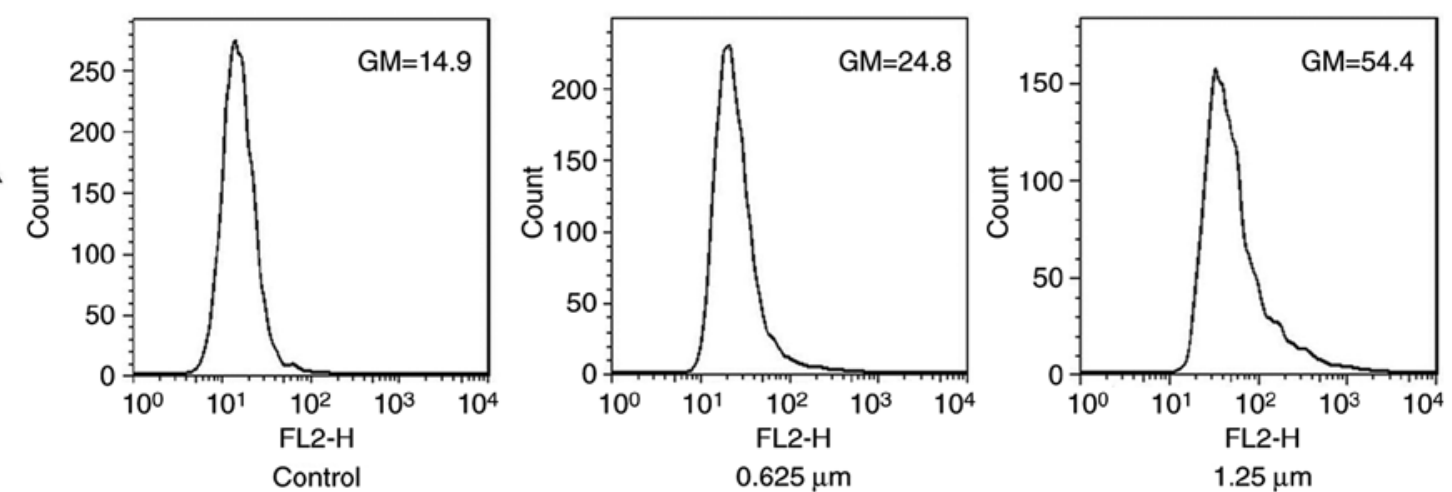

䓂
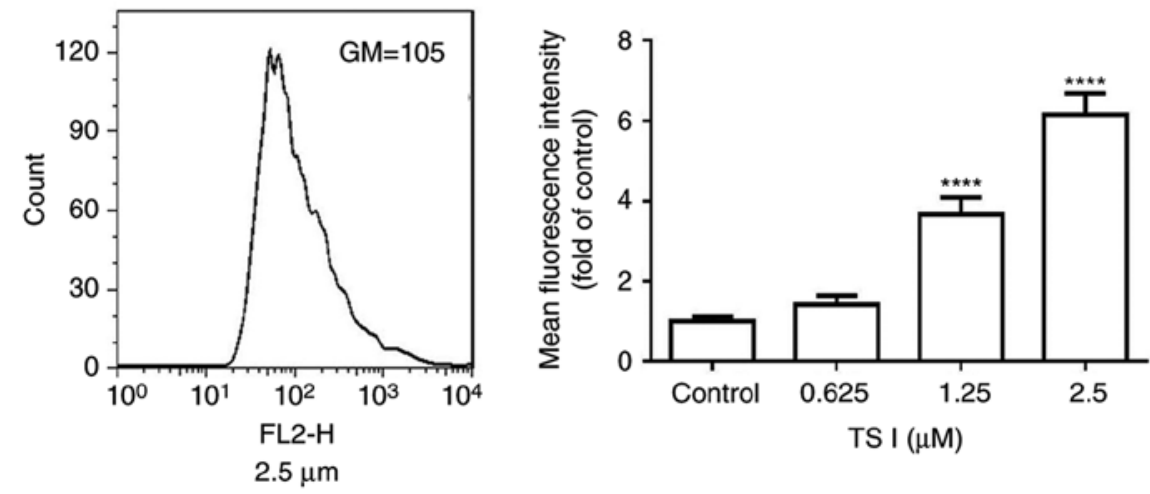

DCFH-DA
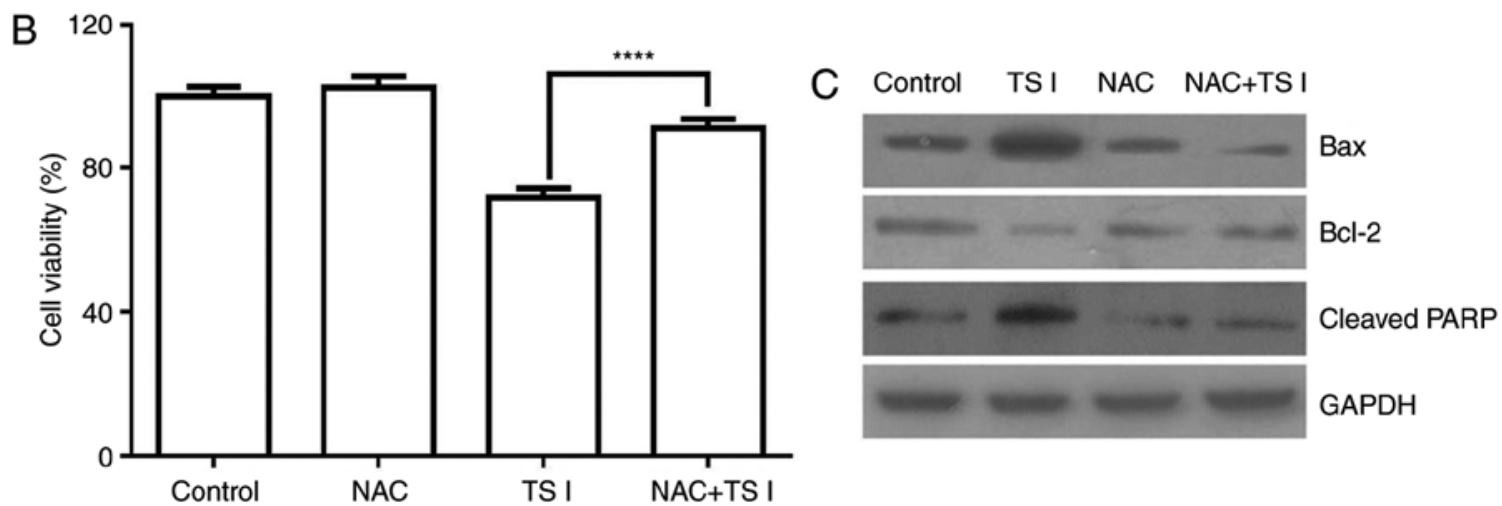

Figure 4. Effects of TS I on intracellular ROS generation in U87 MG cells. (A) Cells were treated with TS I ( $0,0.625,1.25$ and $2.5 \mu$ m) for 12 h, and the levels of ROS were examined using flow cytometry. Bar graphs indicate the quantification of ROS. (B) U87 MG cells were pretreated with NAC (4 mM) for $1 \mathrm{~h}$, followed by treatment with TS I $(1.25 \mu \mathrm{m})$ for $24 \mathrm{~h}$. Cell viability was then detected using a Cell Counting Kit- 8 assay. (C) U87 MG cells were pretreated with NAC ( $4 \mathrm{mM})$ for $1 \mathrm{~h}$, followed by treatment with TS I $(1.25 \mu \mathrm{m})$ for $12 \mathrm{~h}$, and the protein levels of Bcl-2, Bax and cleaved PARP were subsequently assessed using western blot analysis. GAPDH was used as a loading control. ${ }^{* * * *} \mathrm{P}<0.0001$. TS-I, tanshinone I; ROS, reactive oxygen species; NAC, N-acetyl-L-cysteine; Bcl-2, B-cell lymphoma 2; Bax, Bcl-2-associated X protein; PARP, poly(ADP-ribose) polymerase.

cells $(25,26)$. Cell cycle progression is dependent on the activity of cyclin-dependent kinase (CDK) complexes $(27,28)$. The downregulation of cyclin B1 may lead to the inhibition of the cell cycle at the G2/M phase $(29,30)$. It has also been demonstrated that $\mathrm{p} 21$ is a broad-spectrum CDK inhibitor and may promote cell cycle arrest by inhibiting the activity of a number of cyclin-CDK complexes $(31,32)$. Furthermore, a number of studies have revealed that the upregulated expression of p21 plays a key role in $\mathrm{G} 2 / \mathrm{M}$ arrest $(33,34)$. Additionally, it has been reported that TS I-induced inhibition of cyclin A and cyclin B decreases cell cycle progression through the $\mathrm{S}$ and G2/M phases (35). Wang et al (11) revealed that TS I inhibited cell cycle progression by decreasing cyclin B and CDK2 protein levels. The results of the present study demonstrated that TS I upregulated the p21 level and decreased the levels of cyclin B1. These data revealed that TS I caused G2/M arrest by upregulating p21 and downregulating cyclin B1 expression.

Apoptosis is an important physiological process of programmed cell death and serves as an important homeostatic mechanism that balances cell growth and cell death $(36,37)$. The induction of apoptosis in cancer cells is a strategy that may be used in the screening of new anticancer agents (38). A variety of studies have suggested that TS I can induce apoptosis in a variety of human cancer cells. TS I-induced apoptotic death of human breast cancer cells was indicated to 

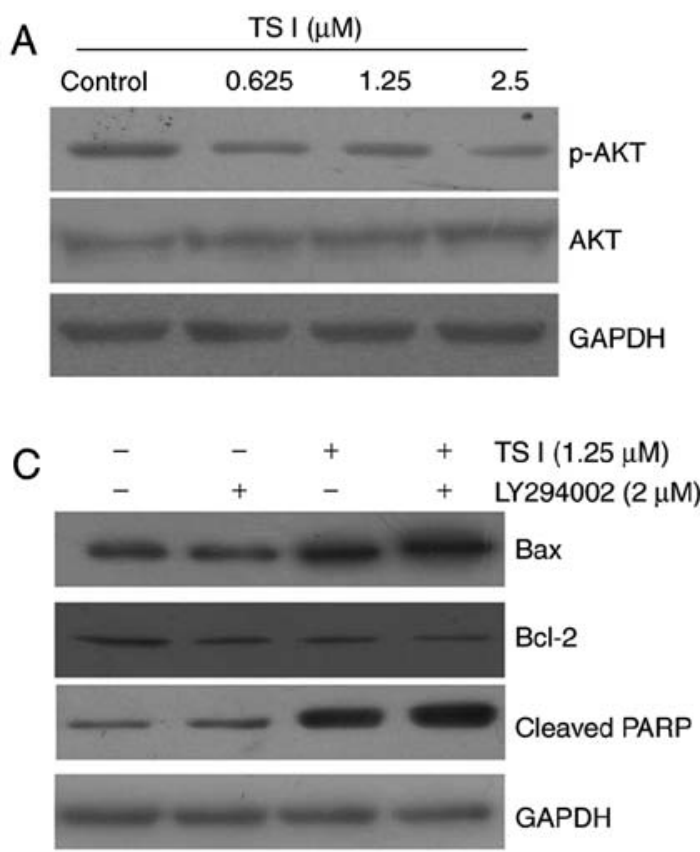
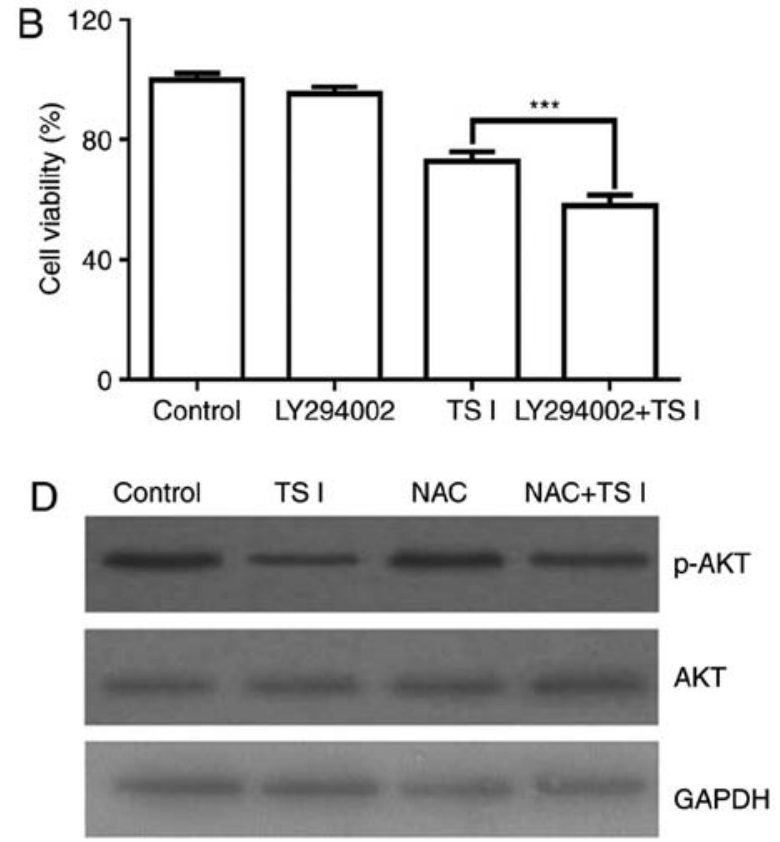

Figure 5. Effects of TS I on the AKT signaling pathway in U87 MG cells. (A) Cells were treated with TS I (0, $0.625,1.25$ and $2.5 \mu$ m) for 24 h, and the expression of GAPDH, total and phosphorylated AKT were detected using western blot analysis. (B) U87 MG cells were pre-treated with LY294002 ( $2 \mu$ m) for $1 \mathrm{~h}$, followed by treatment with TS I $1.25 \mu \mathrm{m}$ for $24 \mathrm{~h}$, and cell viability was subsequently detected using a cell counting kit- 8 assay. (C) U87 MG cells were pre-treated with LY294002 $(2 \mu \mathrm{m})$ for $1 \mathrm{~h}$, followed by treatment with TS I $1.25 \mu \mathrm{m}$ for $12 \mathrm{~h}$, and protein levels of Bcl-2, Bax and cleaved PARP were then assessed using western blot analysis. (D) U87 MG cells were pre-treated with NAC ( $4 \mathrm{mM}$ ) for $1 \mathrm{~h}$, followed by treatment with TS I $1.25 \mu \mathrm{m}$ for $12 \mathrm{~h}$, and the protein levels of GAPDH, total and phosphorylated AKT were then assessed using western blot analysis. GAPDH was used as a loading control. ${ }^{* * *} \mathrm{P}<0.001$; TS-I, tanshinone I; NAC, N-acetyl-L-cysteine; Bcl-2, B-cell lymphoma 2; Bax, Bcl-2-associated X protein; PARP, poly(ADP-ribose) polymerase.

be mediated by the activation of caspase 3 , the downregulation of Bcl-2 and the upregulation of Bax expression (12). In human colon cancer COLO-205 cells, TS I was revealed to promote apoptosis by increasing the expression of Bax and caspase-3 proteins (13). In the present study, treatment with TS I was demonstrated to significantly induce apoptosis by upregulating the expression of cleaved PARP and Bax and downregulating the expression of Bcl-2 in U87 MG cells. These data indicate that apoptosis plays a key role in TS I-induced U87 MG cell death.

AKT kinase, which is a serine/threonine kinase, plays an important role in a number of biological processes, including cell proliferation, differentiation and apoptosis $(39,40)$. A number of studies have demonstrated that natural compounds can induce cancer cell apoptosis through the suppression of the AKT signaling pathway (41-43). Additionally, Wang et al (11) revealed that TS I induced breast cancer cell apoptosis by regulating the $\mathrm{PI} 3 \mathrm{~K} / \mathrm{AKT} / \mathrm{mammalian}$ target of rapamycin signaling pathway. Furthermore, it has been demonstrated that the AKT signaling pathway mediates the mitochondrial apoptotic pathway by increasing the $\mathrm{Bax} / \mathrm{Bcl}-2$ expression ratio and activating PARP (44-46). The results of the present study demonstrated that TS I significantly decreased Bcl-2 and p-AKT protein expression, and significantly increased cleaved PARP protein expression. Furthermore, TS I and LY294002 enhanced the pro-apoptotic effects of TS I. These data suggest that the AKT pathway was associated with apoptosis in U87 MG cells that were treated with TS I. A number of studies have demonstrated that ROS serves as a mediator of apoptosis in a variety of types of therapeutic drug-induced apoptosis $(23,47,48)$.

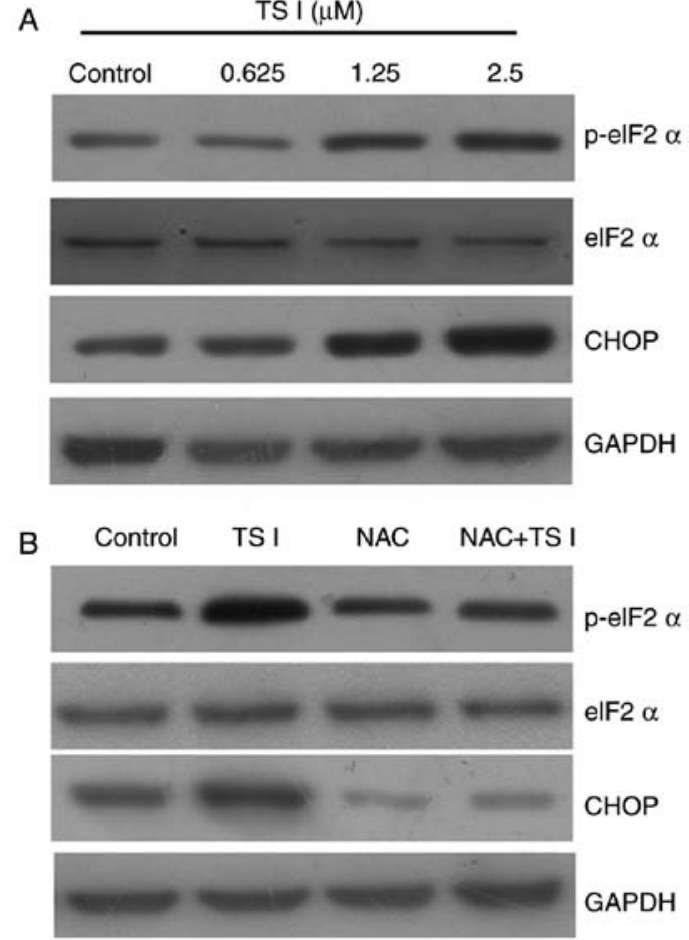

Figure 6. Effects of TS I on the ER stress signaling pathway in U87 MG cells (A) Cells were treated with TS I $(0,0.625,1.25$ and $2.5 \mu \mathrm{m})$ for $12 \mathrm{~h}$, and the expressions of eIF $2 \alpha$, p-eIF $2 \alpha$ and CHOP proteins were detected using western blot analysis. (B) U87 MG cells were pre-treated with NAC (4 mM) for $1 \mathrm{~h}$, followed by treatment with TS I $1.25 \mu \mathrm{m}$ for $12 \mathrm{~h}$, and the protein levels of eIF $2 \alpha$, p-eIF2 $\alpha$ and CHOP were then assessed using western blot analysis. GAPDH was used as a loading control. TS-I, tanshinone I; NAC, $\mathrm{N}$-acetyl-L-cysteine ER, endoplasmic reticulum; eIF2 $\alpha$, eukaryotic initiation factor; $\mathrm{CHOP}, \mathrm{C} / \mathrm{EBP}$ homologous protein. 
A

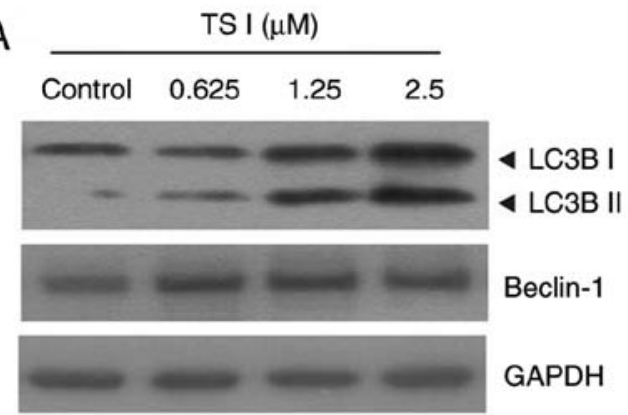

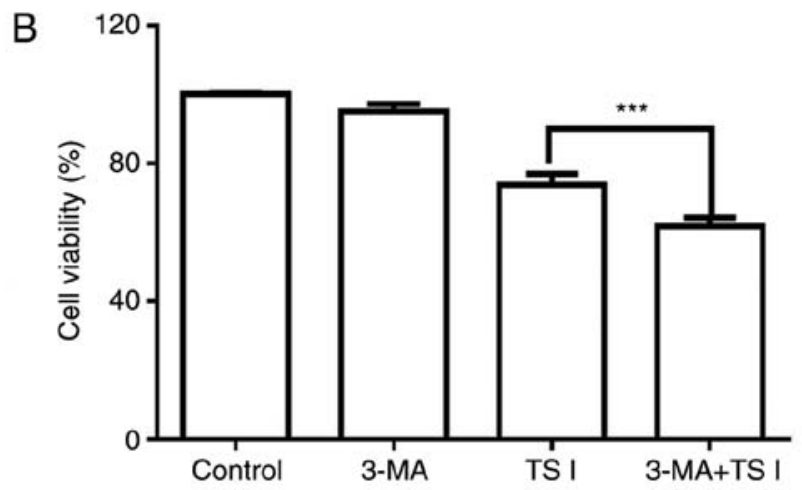

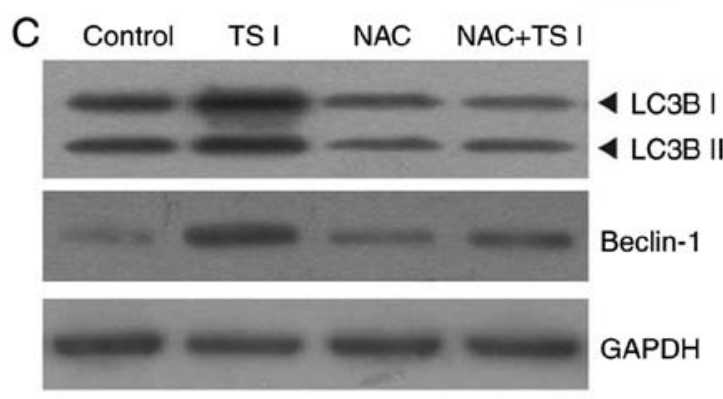

Figure 7. Effects of TS I on cell autophagy in U87 MG cells. (A) Cells were treated with TS I ( $0,0.625,1.25$ and $2.5 \mu$ m) for 12 h, and the expression of LC3B and beclin-1 were detected using western blot analysis. (B) U87 MG cells were pretreated with 3-MA (2 mM) for $1 \mathrm{~h}$, followed by treatment with TS I (1.25 $\mu \mathrm{m})$ for $24 \mathrm{~h}$, and then cell viability was detected using a Cell Counting Kit-8 assay. (C) U87 MG cells were pretreated with 3-MA (2 mM) for 1 h, followed by treatment with TS I $(1.25 \mu \mathrm{m})$ for $12 \mathrm{~h}$, and protein levels of LC3B and beclin-1 were then assayed using western blot analysis. GAPDH was used as a loading control. ${ }^{* * *} \mathrm{P}<0.001$; TS-I, tanshinone I; 3-MA, 3-methyladenine.

In human endometrial carcinoma HEC-1-A cells, TS I has been shown to increase ROS levels (49). In colon cancer cells, TS I has been demonstrated to induce apoptosis via the ROS-mediated p38 signaling pathway (9). In the present study, TS I significantly increased the intracellular levels of ROS in U87 MG cells. Additionally, previous studies have reported that ROS is a potential upstream regulator of PI3K/AKT inactivation, which are key molecules in cell apoptosis $(19,20,50)$. In the present study, NAC was indicated to efficiently reverse the TS I-mediated cell apoptosis and inhibition of AKT phosphorylation in U87 MG cells. These data suggested that ROS may act as a mediator in TS I-induced U87 MG cell apoptosis via the inactivation of the AKT signaling pathway.

It has been widely reported that ER stress may lead to ER dysfunction and the activation of the ER stress-related signaling pathway, which is associated with ROS-mediated cell apoptosis in a variety of cancer types (51-53). TS IIA inhibited human prostate cancer cell proliferation via the induction of ER stress in vitro and in vivo (53). In human lung cancer cells, TS IIA increased TRAIL-induced cell death via the activation of ER stress (54). Consistently with these reports, the results of the present study indicated that TS I increased the expression of the ER stress-related proteins p-eIF $2 \alpha$, ATF4 and CHOP. Furthermore, the downregulation of $\mathrm{CHOP}$ markedly attenuated the inhibition of TS I-induced growth in human glioma U87 MG cells. These data suggested that ER stress may be associated with ROS-mediated TS I-induced cell apoptosis. To further determine the role of ROS in the activation of ER stress induced by TS I, cells were pretreated with NAC. The results revealed that NAC pretreatment significantly attenuated the TS 1-mediated activation of ER stress.
Overall, the results of the present study revealed that TS I promotes apoptosis of U87 MG cells via the ROS-mediated ER stress pathway.

Autophagy can be activated by a variety of different factors, including ROS accumulation and anticancer drugs, and is well-known to play an important role in determining cell fate $(23,55,56)$. In lung cancer $95 \mathrm{D}$ cells, tanshinones induced protective autophagy by increasing ROS production (57). Jing et al (10) also reported that TS I induced protective autophagy in gastric cancer. In the present study, the results demonstrated that autophagy was activated by TS I in human glioma U87 MG cells. Furthermore, pretreatment with 3-MA effectively enhanced the TS I-induced inhibition of U87 MG cells. Additionally, NAC significantly reduced TS I-induced autophagy. Therefore, these results indicate that TS I induces protective autophagy via ROS production in U87 MG cells.

In summary, the results of the present study demonstrated that TS I inhibited the proliferation of human glioma U87 MG cells via the induction of apoptosis and $\mathrm{G} 2 / \mathrm{M}$ cell cycle arrest. TS I was shown to mediate G2/M cell cycle arrest in U87 MG cells by upregulating p21 and downregulating cyclin B1 expression. TS I induced apoptosis by upregulating the expression of cleaved PARP and Bax, and downregulating the expression of Bcl-2. Furthermore, TS I induced apoptosis via the inactivation of the AKT signaling pathway. Therefore, these findings may be an important indication that TS I is a potential anticancer drug candidate that may prove useful in the treatment of human glioma. We aim to further investigate the specificity of TS I against malignant cells in a future study. 


\section{Acknowledgements}

Not applicable.

\section{Funding}

The present study was supported in part by a grant from the National Natural Science Foundation of China (no. 81572485) and the Natural Science Basic Research Plan in Shaanxi Province of China (program no. 2017JQ8037).

\section{Availability of data and materials}

The datasets used and/or analyzed during the present study are available from the corresponding author on reasonable request.

\section{Authors' contributions}

SJ, LC and GS conducted the experiments and analyzed the data. GS made substantial contributions to the design of the present study and prepared the manuscript. LM, CH, TR, FX and ZB performed the western blotting and analyzed the data. All authors have read and approved the final manuscript.

\section{Ethics approval and consent to participate}

Not applicable.

\section{Patient consent for publication}

Not applicable.

\section{Competing interests}

The authors declare that they have no competing interests.

\section{References}

1. Jiang Y and Uhrbom L: On the origin of glioma. Ups J Med Sci 117: 113-121, 2012.

2. Cloughesy TF, Cavenee WK and Mischel PS: Glioblastoma: From molecular pathology to targeted treatment. Annu Rev Pathol 9: 1-25, 2014.

3. Stupp R, Mason WP, van den Bent MJ, Weller M, Fisher B, Taphoorn MJ, Belanger $\mathrm{K}$, Brandes AA, Marosi C, Bogdahn U, et al: Radiotherapy plus concomitant and adjuvant temozolomide for glioblastoma. N Engl J Med 352: 987-996, 2005.

4. Neyns B, Tosoni A, Hwu WJ and Reardon DA: Dose-dense temozolomide regimens: Antitumor activity, toxicity, and immunomodulatory effects. Cancer 116: 2868-2877, 2010

5. Fish JM, Welchons DR, Kim YS, Lee SH, Ho WK and Antzelevitch C: Dimethyl lithospermate B, an extract of Danshen, suppresses arrhythmogenesis associated with the Brugada syndrome. Circulation 113: 1393-1400, 2006.

6. Chang PN, Mao JC, Huang SH, Ning L, Wang ZJ, On T, Duan W and Zhu YZ: Analysis of cardioprotective effects using purified Salvia miltiorrhiza extract on isolated rat hearts. J Pharmacol Sci 101: 245-249, 2006.

7. Tian $\mathrm{XH}$ and $\mathrm{Wu} \mathrm{JH}$ : Tanshinone derivatives: A patent review (January 2006-September 2012). Expert Opin Ther Pat 23: 19-29, 2013.

8. Wang W, Li J, Ding Z, Li Y, Wang J, Chen S and Miao J: Tanshinone I inhibits the growth and metastasis of osteosarcoma via suppressing JAK/STAT3 signalling pathway. J Cell Mol Med 23: 6454-6465, 2019.
9. Kim DH, Shin EA, Kim B, Shim BS and Kim SH: Reactive oxygen species-mediated phosphorylation of p38 signaling is critically involved in apoptotic effect of Tanshinone I in colon cancer cells. Phytother Res 32: 1975-1982, 2018.

10. Jing X, Xu Y, Cheng W, Guo S, Zou Y and He L: Tanshinone I induces apoptosis and pro-survival autophagy in gastric cancers. Cancer Chemother Pharmacol 77: 1171-1181, 2016.

11. Wang L, Wu J, Lu J, Ma R, Sun D and Tang J: Regulation of the cell cycle and PI3K/Akt/mTOR signaling pathway by tanshinone I in human breast cancer cell lines. Mol Med Rep 11: 931-939, 2015.

12. Nizamutdinova IT, Lee GW, Son KH, Jeon SJ, Kang SS, Kim YS, Lee JH, Seo HG, Chang KC and Kim HJ: Tanshinone I effectively induces apoptosis in estrogen receptor-positive (MCF-7) and estrogen receptor-negative (MDA-MB-231) breast cancer cells. Int J Oncol 33: 485-491, 2008.

13. Su CC, Chen GW and Lin JG: Growth inhibition and apoptosis induction by tanshinone I in human colon cancer Colo 205 cells. Int J Mol Med 22: 613-618, 2008.

14. Lu M, Wang C and Wang J: Tanshinone I induces human colorectal cancer cell apoptosis: The potential roles of Aurora A-p53 and survivin-mediated signaling pathways. Int J Oncol 49: 603-610, 2016

15. Gdowski A, Panchoo M, Treuren TV and Basu A: Emerging therapeutics for targeting Akt in cancer. Front Biosci (Landmark Ed) 21: 757-768, 2016.

16. Lien EC, Dibble CC and Toker A: PI3K signaling in cancer: Beyond AKT. Curr Opin Cell Biol 45: 62-71, 2017.

17. Atkins RJ, Dimou J, Paradiso L, Morokoff AP, Kaye AH, Drummond KJ and Hovens CM: Regulation of glycogen synthase kinase-3 beta (GSK-3 $\beta$ ) by the Akt pathway in gliomas. J Clin Neurosci 19: 1558-1563, 2012.

18. Jiang L, Wang C, Lei F, Zhang L, Zhang X, Liu A, Wu G, Zhu J and Song L: miR-93 promotes cell proliferation in gliomas through activation of PI3K/Akt signaling pathway. Oncotarget 6: 8286-8299, 2015.

19. Ji L, Zhong B, Jiang X, Mao F, Liu G, Song B, Wang CY, Jiao Y, Wang JP, Xu ZB, Li X and Zhan B: Actein induces autophagy and apoptosis in human bladder cancer by potentiating ROS/JNK and inhibiting AKT pathways. Oncotarget 8: 112498-112515, 2017.

20. Ahn KI, Choi EO, Kwon DH, HwangBo H, Kim MY, Kim HJ, Ji SY, Hong SH, Jeong JW, Park C, et al: Induction of apoptosis by ethanol extract of Citrus unshiu Markovich peel in human bladder cancer T24 cells through ROS-mediated inactivation of the PI3K/Akt pathway. Biosci Trends 11: 565-573, 2017.

21. Gorrini C, Harris IS and Mak TW: Modulation of oxidative stress as an anticancer strategy. Nat Rev Drug Discov 12: 931-947, 2013.

22. Zhao Q, Liu Y, Zhong J, Bi Y, Liu Y, Ren Z, Li X, Jia J, Yu M and $\mathrm{Yu} \mathrm{X}$ : Pristimerin induces apoptosis and autophagy via activation of ROS/ASK1/JNK pathway in human breast cancer in vitro and in vivo. Cell Death Discov 5: 125, 2019.

23. Liu Y, Kang X, Niu G, He S, Zhang T, Bai Y, Li Y, Hao H, Chen C, Shou $\mathrm{Z}$ and $\mathrm{Li} \mathrm{B}$ : Shikonin induces apoptosis and prosurvival autophagy in human melanoma A375 cells via ROS-mediated ER stress and p38 pathways. Artif Cells Nanomed Biotechnol 47: 626-635, 2019.

24. Yuan X, Wang B, Yang L and Zhang Y: The role of ROS-induced autophagy in hepatocellular carcinoma. Clin Res Hepatol Gastroenterol 42: 306-312, 2018.

25. Vermeulen K, Van Bockstaele DR and Berneman ZN: The cell cycle: A review of regulation, deregulation and therapeutic targets in cancer. Cell Prolif 36: 131-149, 2003.

26. Chao JI, Kuo PC and Hsu TS: Down-regulation of survivin in nitric oxide-induced cell growth inhibition and apoptosis of the human lung carcinoma cells. J Biol Chem 279: 20267-20276, 2004.

27. Zhang J, Su G, Lin Y, Meng W, Lai JKL, Qiao L, Li X and Xie X: Targeting cyclin-dependent kinases in gastrointestinal cancer therapy. Discov Med 27: 27-36, 2019.

28. Bloom J and Cross FR: Multiple levels of cyclin specificity in cell-cycle control. Nat Rev Mol Cell Biol 8: 149-160, 2007.

29. Yu CY, Jerry Teng CL, Hung PS, Cheng CC, Hsu SL, Hwang GY and Tzeng YM: Ovatodiolide isolated from Anisomeles indica induces cell cycle G2/M arrest and apoptosis via a ROS-dependent ATM/ATR signaling pathways. Eur J Pharmacol 819: 16-29, 2017.

30. Lee MH, Cho Y, Kim DH, Woo HJ, Yang JY, Kwon HJ, Yeon MJ, Park M, Kim SH, Moon C, et al: Menadione induces G2/M arrest in gastric cancer cells by down-regulation of CDC25C and proteasome mediated degradation of CDK1 and cyclin B1. Am J Transl Res 8: 5246-5255, 2016. 
31. Stivala LA, Cazzalini O and Prosperi E: The cyclin-dependent kinase inhibitor p21CDKN1A as a target of anti-cancer drugs. Curr Cancer Drug Targets 12: 85-96, 2012.

32. Starostina NG and Kipreos ET: Multiple degradation pathways regulate versatile CIP/KIP CDK inhibitors. Trends Cell Biol 22: 33-41, 2012.

33. Gong FR, Wu MY, Shen M, Zhi Q, Xu ZK, Wang R, Wang WJ, Zong Y, Li ZL, Wu Y, et al: PP2A inhibitors arrest G2/M transition through JNK/Sp1- dependent down-regulation of CDK1 and autophagy-dependent up-regulation of $\mathrm{p} 21$. Oncotarget 6 : 18469-18483, 2015.

34. Wang LH, Jiang XR, Chen GL, Guo W, Zhang JY, Cui LJ, Li HH, Li M, Liu X, Yang JY and Wu CF: Anti-tumor activity of SL4 against breast cancer cells: Induction of $\mathrm{G}_{2} / \mathrm{M}$ arrest through modulation of the MAPK-dependent $\mathrm{p} 21$ signaling pathway. Sci Rep 6: 36486, 2016.

35. Tung YT, Chen HL, Lee CY, Chou YC, Lee PY, Tsai HC, Lin YL and Chen CM: Active component of danshen (Salvia miltiorrhiza Bunge), Tanshinone I, attenuates lung tumorigenesis via inhibitions of VEGF, cyclin A, and cyclin B expressions. Evid Based Complement Alternat Med 2013: 319247, 2013.

36. Liu G, Pei F, Yang F, Li L, Amin AD, Liu S, Buchan JR and Cho WC: Role of autophagy and apoptosis in non-small-cell lung cancer. Int J Mol Sci 18: E367, 2017.

37. Croce CM and Reed JC: Finally, an apoptosis-targeting therapeutic for cancer. Cancer Res 76: 5914-5920, 2016.

38. Cheng YL, Lee SC, Lin SZ, Chang WL, Chen YL, Tsai NM, Liu YC, Tzao C, Yu DS and Harn HJ: Anti-proliferative activity of Bupleurum scrozonerifolium in A549 human lung cancer cells in vitro and in vivo. Cancer Lett 222: 183-193, 2005.

39. Manning BD and Toker A: AKT/PKB signaling: Navigating the network. Cell 169: 381-405, 2017.

40. Spangle JM, Roberts TM and Zhao JJ: The emerging role of PI3K/AKT-mediated epigenetic regulation in cancer. Biochim Biophys Acta Rev Cancer 1868: 123-131, 2017.

41. Kello M, Kulikova L, Vaskova J, Nagyova A and Mojzis J: Fruit peel polyphenolic extract-induced apoptosis in human breast cancer cells is associated with ROS production and modulation of p38MAPK/Erk1/2 and the akt signaling pathway. Nutr Cancer 69: 920-931, 2017.

42. Jovaisas E, Koch MA, Schafer A, Stauber M and Lowenthal D: LAV/HTLV-III in 20-week fetus. Lancet 2: 1129, 1985.

43. Moeinifard M,Hassan ZM, Fallahian F, Hamzeloo-Moghadam M and Taghikhani M: Britannin induces apoptosis through AKT-FOXO1 pathway in human pancreatic cancer cells. Biomed Pharmacother 94: 1101-1110, 2017.

44. Yaoi X, Lu B, Lu C, Bai Q, Yan D and Xu H: Taraxerol induces cell apoptosis through A mitochondria-mediated pathway in HeLa cells. Cell J 19: 512-519, 2017.

45. Li C, Wang Y, Wang C, Yi X, Li M and He X: Anticancer activities of harmine by inducing a pro-death autophagy and apoptosis in human gastric cancer cells. Phytomedicine 28: 10-18, 2017.

46. Kim MJ, Kwon SB, Kim MS, Jin SW, Ryu HW, Oh SR and Yoon DY: Trifolin induces apoptosis via extrinsic and intrinsic pathways in the NCI-H460 human non-small cell lung-cancer cell line. Phytomedicine 23: 998-1004, 2016.
47. Thiagarajan S, Arapoc DJ, Husna Shafie N, Keong YY, Bahari H, Adam $Z$ and Ei T: Momordica charantia (Indian and Chinese Bitter Melon) extracts inducing apoptosis in human lung cancer cell line A549 via ROS-mediated mitochodria injury. Evid Based Complement Alternat Med 2019: 2821597, 2019.

48. N B, Chandrashekar KR, Prabhu A and Rekha PD: Tetrandrine isolated from Cyclea peltata induces cytotoxicity and apoptosis through ROS and caspase pathways in breast and pancreatic cancer cells. In Vitro Cell Dev Biol Anim 55: 331-340, 2019.

49. Li Q, Zhang J, Liang Y, Mu W, Hou X, Ma X and Cao Q: Tanshinone 1 exhibits anticancer effects in human endometrial carcinoma HEC-1-A cells via mitochondrial mediated apoptosis, cell cycle arrest and inhibition of JAK/STAT signalling pathway. J BUON 23: 1092-1096, 2018.

50. Song X, Wang Z, Liang H, Zhang W, Ye Y, Li H, Hu Y, Zhang Y, Weng H, Lu J, et al: Dioscin induces gallbladder cancer apoptosis by inhibiting ROS-mediated PI3K/AKT signalling. Int J Biol Sci 13: 782-793, 2017.

51. Huang H, Xie H, Pan Y, Zheng K, Xia Y and Chen W: Plumbagin triggers ER stress-mediated apoptosis in prostate cancer cells via induction of ROS. Cell Physiol Biochem 45: 267-280, 2018.

52. Kim SM, Lee HM, Hwang KA and Choi KC: Benzo(a)pyrene induced cell cycle arrest and apoptosis in human choriocarcinoma cancer cells through reactive oxygen species-induced endoplasmic reticulum-stress pathway. Food Chem Toxicol 107: 339-348, 2017.

53. Yang Y, Zhang Y, Wang L and Lee S: Levistolide a induces apoptosis via ROS-mediated ER stress pathway in colon cancer cells. Cell Physiol Biochem 42: 929-938, 2017.

54. Zhao D, Tong L, Zhang L, Li H, Wan Y and Zhang T: Tanshinone II A stabilizes vulnerable plaques by suppressing RAGE signaling and NF- $\kappa \mathrm{B}$ activation in apolipoprotein-E-deficient mice. Mol Med Rep 14: 4983-4990, 2016.

55. Geng YD, Zhang L, Wang GY, Feng XJ, Chen ZL, Jiang L and Shen AZ: Xanthatin mediates $\mathrm{G}_{2} / \mathrm{M}$ cell cycle arrest, autophagy and apoptosis via ROS/XIAP signaling in human colon cancer cells. Nat Prod Res 27: 1-5, Dec 27, 2018 (Epub ahead of print).

56. Wang B, Zhou TY, Nie CH, Wan DL and Zheng SS: Bigelovin, a sesquiterpene lactone, suppresses tumor growth through inducing apoptosis and autophagy via the inhibition of mTOR pathway regulated by ROS generation in liver cancer. Biochem Biophys Res Commun 499: 156-163, 2018.

57. Gao H, Sun W, Zhao W, Hao W, Leung $\mathrm{CH}, \mathrm{Lu} \mathrm{J}$ and Chen $\mathrm{X}$ Total tanshinones-induced apoptosis and autophagy via reactive oxygen species in lung cancer 95D cells. Am J Chin Med 43: $1265-1279,2015$

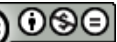

This work is licensed under a Creative Commons Attribution-NonCommercial-NoDerivatives 4.0 International (CC BY-NC-ND 4.0) License. 